\title{
Statement by a Working Group conceived by the Polish National Consultants in Cardiology and Neurology addressing the use of implantable cardiac monitors in patients after ischaemic embolic stroke of undetermined source
}

Michał M. Farkowski ${ }^{1}$, Michał A. Karliński², Jarosław Kaźmierczak ${ }^{3}$, Piotr Kułakowski ${ }^{4}$, Przemysław Mitkowski ${ }^{5}$, Paweł Ptaszyński ${ }^{6}$, Danuta Ryglewicz ${ }^{2}$, Jarosław Sławek ${ }^{7}$,

\author{
Agnieszka Słowik ${ }^{8}$, Maciej Sterliński ${ }^{1}$ \\ ${ }^{1}$ Institute of Cardiology, Warsaw, Poland \\ ${ }^{2}$ Institute of Psychiatry and Neurology, Warsaw, Poland \\ ${ }^{3}$ Pomeranian Medical University, Szczecin, Poland \\ ${ }^{4}$ Postgraduate Medical Education Center, Warsaw, Poland \\ ${ }^{5}$ Poznan University of Medical Sciences, Poland \\ ${ }^{6}$ Medical University of Lodz, Poland \\ ${ }^{7}$ Medical University of Gdansk, Poland
}

${ }^{8}$ Jagiellonian University Medical College, Krakow, Poland

\begin{abstract}
Introduction. Stroke remains one of the main causes of death and the most common cause of long-term disability in adults. Embolic strokes of undetermined source (ESUS) amount to a significant proportion of all ischaemic strokes. Detection of atrial fibrillation (AF) in this group of patients would allow for a major therapeutic decision to switch from antiplatelets to oral anticoagulants and therefore significantly reduce the risk of recurrence.

State of the Art. Current technology allows long-term continuous ECG monitoring with different systems, including implantable cardiac monitors (ICM). However, in Poland lack of reimbursement does not allow their use in everyday clinical practice. Clinical Implications. This is a statement by a Working Group conceived by the Polish National Consultants in Cardiology and Neurology addressing the use of ICM in patients after ischaemic embolic strokes of undetermined source. The aim was to develop reasonable and comprehensive guidance on how to select and manage candidates for ICM in order to obtain the maximum benefit for Polish public health.

Future Directions. This expert opinion is not intended as a guideline but it provides advice as to how to optimise the potential use of ICM in patients after ESUS in the Polish setting.

Key words: acute ischaemic stroke, embolic stroke of undetermined source, atrial fibrillation, ECG monitoring (Neurol Neurochir Pol 2019; 53 (3): 181-189)
\end{abstract}

Address for correspondence: Michał A. Karlinski, Institute of Psychiatry and Neurology, Warsaw, Poland, e-mail: mkarlinski@ipin.edu.pl 


\section{Therapeutic problem}

Despite continuous progress in prevention and treatment observed over recent decades, stroke remains one of the main causes of death, and the most common cause of long-term disability, in adults in developed countries $[1,2]$.

In about $80 \%$ of cases stroke is ischaemic [2]. According to the map of health needs related to hospital treatment in Poland developed by the Ministry of Health, ischaemic stroke was the main cause of 64,400 hospital stays in Polish stroke units in 2016 [3]. Only about $9 \%$ of patients with the principal diagnosis of acute ischaemic stroke were treated in non-stroke departments [3].

The mortality rate in Polish patients after ischaemic stroke was $14.5 \%$ within 30 days, $21.5 \%$ within 90 days, and reached $30.2 \%$ at 12 months [3].

In terms of aetiology, stroke is a heterogeneous condition. Therefore, in order to implement optimal secondary prevention, it is necessary to individually identify its most probable cause in each patient. According to the generally applicable TOAST (Trial of Org 10172 in Acute Stroke Treatment) classification, the main causes of ischaemic stroke may be [4]:

- Large artery atherosclerosis (about $25 \%$ of ischaemic strokes worldwide [5], and 18\% in Poland [6]; 5-year recurrence $23 \%, 5$-year risk of death $31 \%$ [7]). Diagnosed if there is $\geq 50 \%$ stenosis or occlusion of a large artery or its branches supplying the infarct area with no evidence of a cardiac source of embolism.

- Cardioembolism (about 20\% of ischaemic strokes worldwide [5], and 28\% in Poland [6]; 5-year recurrence 25\%, 5 -year risk of death 65\% [7]). Diagnosed if there is at least one potential source of embolic material (e.g. atrial fibrillation, prosthetic cardiac valve, left ventricular or atrial thrombus, sick sinus syndrome, recent myocardial infarction, dilated cardiomyopathy, akinetic left ventricular segment, atrial myxoma, infective endocarditis) with no advanced atherosclerosis of the supplying arteries.

- Small vessel disease (about 25\% of ischaemic strokes worldwide [5], and 12\% in Poland [6]; 5-year recurrence $20 \%, 5$-year risk of death about 20\% [7]). Diagnosed if there is no clinical evidence of cerebral cortical dysfunction and no evident infarct focus or a relevant brain stem lesion or a relevant subcortical lesion measures $<1.5 \mathrm{~cm}$ (so called lacunar lesion).

- Other determined causes (about $5 \%$ of ischaemic strokes worldwide [5], about $4 \%$ in Poland). Most frequently diagnosed in the presence of nonatherosclerotic vasculopathies (e.g. dissection, fibrous dysplasia, vasculitis, moyamoya disease) or hypercoagulable states.

- No clear cause or more than one probable cause (about $25 \%$ of ischaemic strokes worldwide [5], and up to $39 \%$ of ischaemic strokes in Poland [6]; 5-year recurrence 23\%, 5-year risk of death $25 \%$ [7]).
Identification of a cardioembolism is of primary importance for secondary prevention. Randomised studies have not revealed any clear benefits of using anti-platelet agents in this group, which are an implicit standard of treatment of each ischaemic stroke in the acute phase [8]. However, long term treatment with vitamin $\mathrm{K}$ antagonists is known to reduce the relative risk of another ischaemic stroke by 64\% (NNT 12 in 1 year) [8]. A similar or even higher efficacy in preventing stroke compared to warfarin, together with a superior safety profile, has been shown for novel oral anticoagulants (NOAC) $[9,10]$. Moreover, a subsequent ischaemic stroke which occurs despite treatment with an oral anticoagulant is milder and has more favourable outcome [11].

Therefore, cardiological diagnostic workup, especially screening for cardiac arrhythmias, is one of the key elements of a hospital stay in the stroke unit [12]. A positive history of atrial fibrillation or its identification after ischaemic stroke is a clear indication for implementation of oral anticoagulation, preferably with the use of a NOAC [13].

Research data suggests that a major proportion of strokes of unknown aetiology, so called 'cryptogenic' strokes, have in fact an embolic origin [5]. For this reason, in 2014, a new subtype of cryptogenic stroke was introduced: embolic strokes of undetermined source (ESUS) [5]. The diagnosis of ESUS requires combination of all following criteria:

- Non-lacunar ischaemic lesion responsible for the symptoms detected by computed tomography (CT) or magnetic resonance imaging (MRI)

- Absence of extracranial or intracranial atherosclerosis causing $\geq 50 \%$ luminal stenosis in arteries supplying the area of ischaemia (ultrasound, angio-CT or angio-MRI)

- No major risk cardioembolic source of embolism (12-lead ECG, at least 24-hour Holter ECG monitoring and transthoracic echocardiography)

- No other specific cause of stroke identified

It is estimated that 9-25\% of patients with acute ischaemic stroke meet the criteria of ESUS [6, 14]. Compared to the other types of ischaemic stroke, patients with ESUS are younger, have milder neurological deficits, and a better chance of remaining independent in everyday activities $[6,14]$. Therefore, in this group of patients optimal and intense secondary prevention is especially justified.

The results of a prospective analysis of patients hospitalised due to acute stroke at the Institute of Psychiatry and Neurology between 2001 and 2015 show that the real proportion of ESUS in Poland is probably between $10 \%$ and $20 \%$ of all acute ischaemic strokes [6].

With regard to recurrence (3-6\% within one year) and the potential therapeutic benefit of safe anticoagulation with NOAC, two randomised clinical trials were conducted to evaluate the safety and efficacy of long term treatment with rivaroxaban (NAVIGATE ESUS, terminated prematurely in $2017, \mathrm{~N}=7,213$ ) or dabigatran (RE-SPECT ESUS, 
completed in 2018, $\mathrm{N}=5,390$ ) compared to standard dose aspirin [14]. Unfortunately, neither of the studies showed NOAC superiority in preventing strokes $[15,16]$ (World Stroke Conference 2018). Moreover, the use of rivaroxaban was associated with an increased risk of haemorrhagic complications [16].

As a consequence, it must be recognised that the current concept of ESUS has proved to be clinically impractical. Post hoc analyses of the abovementioned trials are likely to provide information as to which ESUS subpopulations might potentially benefit from oral anticoagulation, based on additional demographic, clinical, imaging and electrophysiological criteria. Nevertheless, the hypotheses generated in this way will have to be verified in further randomised trials. Considering the duration of such potential trials, new solutions may appear no sooner than in a few years.

Therefore, it is reasonable to subject patients currently classified as having ESUS based on standard diagnostic criteria to further diagnostic workup. Due to the potential therapeutic implications, targeting confirmation of a suspected cardioembolism seems to be of particular importance. This will allow the identification of patients with a potentially higher risk of recurrence than the average for the whole ESUS group, and subsequently reduce this risk by the introduction of an oral anticoagulant.

It is desirable that ECG monitoring initiated shortly after ESUS be prolonged, especially in the subpopulation with additional signs of atrial disease [17-22]. Supplementary diagnostic procedures should be initiated as soon as possible following the occurrence of stroke, in order to maximise the benefits of potentially indicated anticoagulation. A delayed diagnosis of atrial fibrillation after the first ischaemic stroke is related to a 1.5-fold higher risk of recurrence [23]. It also needs to be emphasised that the curves of survival free of another stroke diverge already in the first year of follow-up, later becoming almost parallel [23].

\section{Standard procedures in the acute phase of ischaemic stroke}

Acute stroke is a life-threatening condition, and as such requires urgent admission to a stroke unit. The diagnosis of stroke is based on symptoms and clinical signs with the support of brain imaging. In Poland, the hospital stay of an acute stroke patient lasts in most cases eight days or longer. This time is fully sufficient to conduct the standard diagnostic process recommended by guidelines, including the guidelines from the Section of Vascular Diseases at the Polish Neurological Society $[12,24,25]$ :

- Brain imaging: CT (usually), CT and MRI (sometimes), or only MRI (rarely); performed at least once on admission to hospital; allows a determination to be made as to whether the stroke is ischaemic or haemorrhagic; it can reveal an acute lesion, silent brain infarcts, and signs of small vessel disease

- Vascular imaging: carotid ultrasound (always), transcranial Doppler (usually), CT angiography or MRI angiography (sometimes additionally); performed to assess atherosclerosis and confirm or exclude other less common vascular pathologies

- Screening for cardiac arrhythmias: resting ECG (at least once), monitoring of heart rate with a cardiomonitor for at least 24 hours (almost always), 24-hour Holter monitoring (usually); may detect previously undiagnosed atrial fibrillation

- Echocardiography: transthoracic echocardiography (usually), transoesophageal echocardiography (as a supplementary examination is some cases); may detect source of cardiac embolism

Reperfusion therapy is the gold standard of treatment in all eligible cases. Intravenous thrombolysis is available at all Polish stroke units and is fully reimbursed. Currently, such treatment is used in about $13 \%$ of patients. Mechanical thrombectomy as a therapy supporting thrombolytic treatment, or as the therapy of choice in patients not eligible to thrombolysis, is indicated for strokes caused by occlusion or critical stenosis. In Poland it is not reimbursed and is therefore used in less than $1 \%$ of patients. A pilot project launched by the Ministry of Health in January 2019 should enable reasonable nationwide access to thrombectomy within the next few years.

All acute patients who are not eligible for reperfusion therapy should receive anti-platelet therapy (usually 150 $300 \mathrm{mg}$ of aspirin, or sometimes a combination of aspirin and clopidogrel). Aspirin is also initiated 24 hours after reperfusion therapy $[12,24,25]$.

After the acute phase, long-term treatment with aspirin $75-150 \mathrm{mg}$ is maintained unless the stroke has been considered to be cardioembolic. In such cases, aspirin should be replaced with an oral anticoagulant as soon as it is safe for the patient $[12,24-26]$.

\section{Atrial fibrillation and ischaemic stroke}

According to the guidelines, atrial fibrillation (AF) is diagnosed on an electrocardiogram showing completely irregular $\mathrm{R}-\mathrm{R}$ intervals with the absence of marked $\mathrm{P}$ waves, lasting at least $30 \mathrm{~s}[13,27]$. AF is the most common arrhythmia in the population. Its prevalence in the general population is about $1-2 \%$ of patients on average. In selected risk groups (e.g. old age, ESUS, numerous general and cardiological diseases), this proportion is much higher $[13,27]$.

AF aetiology is believed to be multifactorial. It entails genetic conditioning, as well as comorbidities and lifestyle [27].

AF may become manifest with general weakness, the sensation of skipping beats, reduced exercise tolerance, chest discomfort, sleeping disorders or anxiety. Basic AF therapy 
involves a strategy of rhythm control or ventricular rate control [13]. AF may result in serious complications leading to death or significant disability: cardiac insufficiency, ischaemic stroke or peripheral embolism [13]. The basis of primary and secondary prevention of stroke is oral anticoagulant therapy (OAT). Each patient should undergo risk stratification regarding vascular events with the use of a $\mathrm{CHA}_{2} \mathrm{DS}_{2}$-VASc scale, and if a score of 2 or higher is achieved - oral anticoagulation should be introduced [13]. It must be remembered that a history of ischaemic stroke adds 2 points to the total $\mathrm{CHA}_{2} \mathrm{DS}_{2}$-VASc score [13]. This means in practice that oral anticoagulation is indicated for every patient with a history of atrial fibrillation and ischaemic stroke, regardless of the time of its detection.

\section{ECG monitoring}

Due to the potentially serious embolic consequences of AF and the possibility of their effective prevention, all scientific societies and expert groups find it justified to screen for AF patients with a high risk of stroke [13,27]. ESUS patients also belong in the group of high risk of both AF and recurrent stroke. Therefore prolonged ECG monitoring after ESUS is recommended both during the stroke unit stay and in the outpatient setting.

Monitoring may be non-invasive (e.g. prolonged Holter monitoring, external arrhythmia recorders, electronic devices) or invasive, in the form of an implantable cardiac monitor (ICM) [27].

Clinical trials have shown that the longer the monitoring, the greater the chance of detecting AF. In CRYSTAL-AF, the use of ICM in patients after cryptogenic stroke resulted in diagnosing AF in an additional $7.5 \%$ of patients $(8.9 \%$ vs $1.4 \%)$ within six months, in an additional $10.4 \%$ (12.4\% vs $2.0 \%)$ within 12 months, and in an additional $27.0 \%$ (30.0\% vs $3.0 \%)$ within three full years of follow-up [28, 29]. In another clinical trial, during a one year follow-up, ICM implantation yielded an additional $15.3 \%$ (17\% vs 1.7\%) AF diagnoses compared to 7-day Holter monitoring [30]. Unfortunately, there are no randomised studies confirming the clinical benefits of anticoagulation therapy in AF diagnosis made with the use of ICM. However, there are no reasons to believe that they will significantly differ from the benefits experienced by patients with AF diagnosed with the use of standard ECG monitoring.

In current clinical practice, prolonged ECG monitoring after a stroke is rare, and qualification for such monitoring is highly individualised [19]. Such practice results from limited financial and human resources and definitely brings no benefit on the level of public health [19]. On the other hand, the high direct cost of prolonged ECG monitoring require the proper selection of patients to maximise the proportion of screenings yielding positive results.

Currently, Polish patients have very limited access to long-term ECG monitoring. ICM implantation and follow-up visits are not reimbursed, and the National Health Fund does not account for the number of days of Holter ECG monitoring, nor does it provide separate financing of external or implantable arrhythmia recorders.

Considering these limitations, and the uncertainty about long-term benefit, it is suggested that only patients with the highest risk of AF occurrence should be referred for such diagnostic workup [18]. Observational studies consistently point to older age as the main risk factor of the occurrence/ recognition of AF after cryptogenic stroke [20].

Currently there are three initially validated scoring systems used to stratify the risk of occurrence/recognition of AF in patients scheduled for long-term ECG monitoring after ESUS that are worth mentioning. These are: MrWALLETS (from -2 to 5 points) [31], HAVOC (from 0 to 14 points) [32], and Brown ESUS-AF score (from 0 to 4 points) [33]. According to a group of experts from the German Cardiac Society and the German Stroke Society there are several predictors of AF in patients after ischaemic stroke, including age $\geq 75$ years, left atrial diameter $>45 \mathrm{~mm}$, detection of supraventricular arrhythmias and increased serum BNP or NT-proBNP level [18]. In contrast, age $<60$ years, left atrial diameter $<40 \mathrm{~mm}$, low number of supraventricular arrhythmias and low serum BNP or NT-proBNP level suggest a low probability of detecting AF [18].

This set of variables overlaps with the Brown ESUS-AF score (age 65-74 years $=1$ point, age $\geq 75$ years $=2$ points, moderate or severe left atrial enlargement $=2$ points). The Brown ESUS-AF score has been developed based on the analysis of 296 ESUS patients subjected to prolonged ECG monitoring (initial 30-day external monitoring followed by optional ICM implantation). Atrial fibrillation was detected in $21 \%$ of patients in a subgroup scoring 2 points (sensitivity $63 \%$, specificity $71 \%$ ), in $22 \%$ of patients scoring 3 points, and in $56 \%$ of patients scoring 4 points [33].

In our opinion, ICM implantation is especially justified in two groups of patients after an ESUS that did not result in a permanent loss of independence in performing daily activities:

- Age 65-74 years with left atrial enlargement $\geq 45 \mathrm{~mm}$

- Age $\geq 75$ years with left atrial enlargement $>40 \mathrm{~mm}$

It may be assumed that after such selection ICM monitoring should result in the detection of AF in about $30 \%$ of patients (similarly to the group with $\geq 2$ points in the Brown ESUS-AF score).

\section{Clinical aspects of the use of ICM}

Modern cardiac implantable electronic devices (CIED: cardiac pacemakers, cardioverter defibrillators, resynchronisation therapy systems), if they enable direct recording of atrial electrical activity, are the best model for identifying supraventricular tachyarrhythmias, since they can do it continuously, 24 hours a day. The system should allow for an 
intracardiac electrogram (IEGM). Based only on the criterion of atrial electrical activity, episodes with a rate exceeding $160-170$ beats/min are usually considered to be atrial high rate episodes (AHRE). Such episodes may include various supraventricular tachycardias, not only episodes of atrial flutter/fibrillation. Therefore, confirmation of the latter is only possible via a careful analysis of IEGM. It must be emphasised that CIEDs are implanted based on a set of well-described indications, and a diagnosis of AHRE is just a side effect of the primary function of the device.

The 2016 guidelines of the European Society of Cardiology on atrial fibrillation recommend (as class I indications) a regular CIED memory interrogation in order to identify AHRE [13]. Patients with diagnosed AHRE should undergo further ECG monitoring in order to document potential AF episodes before the onset of oral anticoagulant therapy, while IEGM recording is enough to document the arrhythmia. The same recommendations for patients after stroke suggest the consideration of additional (more than 72 hours) long-term ECG monitoring with the use of non-invasive ECG recorders or ICM in order to document AF. Implantable cardiac monitors are small devices [mm] 45-88.4 × 7-15.2 x 3-6.2, depending on the manufacturer and model. The recorders are implanted subcutaneously in the parasternal region, in the 4th intercostal space. The battery allows operation of the device for $2-4$ years. The implantation procedure is minimally invasive and performed under local anaesthesia, in the setting of an operating room or treatment room, and is characterised by $100 \%$ efficacy. The device is oriented to achieve the highest possible amplitude of ventricular complexes, usually at a 45 degree angle to the sternal long axis, along the sternum or in any other position between the above two.

Another element of the system is the possibility of telemonitoring. A special modem enables automatic transmission of arrhythmic episodes to a dedicated server. The episodes are available for a physician taking care of the patient within 24 hours of their occurrence. ICMs record only ventricular electrical activity (QRS complexes), so episodes of atrial fibrillation may be suspected only thanks to special algorithms which assess irregularities of R-R intervals on the basis of the analysis of Lorentz plots. Detection of atrial fibrillation is possible when the arrhythmia lasts for at least two minutes. The algorithms detecting atrial fibrillation based on the analysis of changes in R-R intervals are also available in some models of single chamber cardioverter defibrillators. Basic technical data of particular ICMs are presented in Table 1.

Device interrogation (including device memory) is usually conducted every 3-12 months in the device control clinic or by means of remote control (based on the telemonitoring technology) with the same time intervals as controls in the clinic. In the latter case, it is recommended that once a year the interrogation is performed during a visit to the clinic. As previously mentioned, the remote control of devices enables not only automatic reporting at pre-programmed time intervals, but also immediate transmission of information about a significant clinical event (e.g. episode of atrial fibrillation) within 24 hours of its occurrence.

Asymptomatic episodes of atrial fibrillation are much more frequent than symptomatic ones. In a study by Orlow et al., which included 427 patients with an implanted pacemaker in accordance with applicable indications, the risk of an atrial high rate episode after two years of follow-up was 53.8\% and $88.6 \%$ in a subgroup without and with a history of supraventricular tachyarrhythmias, respectively [34]. Moreover, the episodes were asymptomatic in these groups in $94.7 \%$ and $75.3 \%$ of cases, respectively. The symptoms reported by patients were not consistent with arrhythmia episodes in most of the enrolled patients. Importantly, as much as $93 \%$ of AHREs were, in fact, supraventricular tachyarrhythmias and not AFs. In another study involving patients with an implanted CIED, it was found that as much as $95 \%$ of AF episodes are asymptomatic [35]. The percentage of correct AF diagnoses based on the analysis of AHRE in the ICM memory increases with the episode duration: starting from $83 \%$ for episodes $>6$ minutes up to $97 \%$ for episodes $>6$ hours [34]. False positive AHRE diagnoses are usually caused by episodes of: repetitive non-reentrant ventriculo-atrial synchrony (RNRVAS) - 80.4\%, R-wave oversensing on the atrial lead $-7.5 \%$, interference $-6.9 \%$, and other factors $-5.2 \%$.

A subanalysis of the CRYSTAL-AF trial, which included patients after ischaemic stroke with ICM, compared the sensitivity of detecting AF episodes between ICM and other ECG recording methods [36]. Compared to ICM, one 24-hour Holter examination showed a sensitivity of $1.3 \%$, and a continuous 30-day examination 22.8\%. With a 24-hour Holter examination repeated every three months this figure was $3.1 \%$, and with a 7 -day examination it was $20.8 \%$. It is apparent that compared to an AF diagnosis based on implantable episode recorders, all other analysed methods of ECG registration allow detection of only one in every four AF episodes.

The latest algorithms of atrial fibrillation implemented in ICMs are characterised by over $99 \%$ sensitivity and a positive predictive value of $95 \%$ [37].

\section{Optimal patient pathway for ICM implantation}

To ensure early detection and quick therapeutic decision after the occurrence of clinically significant arrhythmia the process of qualification, implantation and outpatient follow-up needs to be standardised.

a) Tasks of the stroke centre

- Identification of an ESUS patient eligible for ICM implantation

- Brief explanation of the whole procedure and obtaining patient's initial consent to a possible ICM implantation

- Initiation of the process of final qualification for ICM implantation at the collaborating cardiological centre 
Table 1. Basic technical data of selected implantable arrhythmic episode recorders

\begin{tabular}{lccc} 
Manufacturer & Abbott & Biotronik & Medtronic \\
\hline Model & Confirm Rx $x^{\text {Tw1 }}$ DM3500 & Biomonitor 2 & $\begin{array}{c}\text { Reveal LINQ } \\
\text { LNQ1 }\end{array}$ \\
Dimensions (mm): length x width x thickness & $49 \times 9 \times 3$ & $88.4 \times 15.2 \times 6.2$ & $45 \times 7 \times 4$ \\
Volume $\left(\mathrm{cm}^{3}\right.$ ) & 1.4 & 5 & 1.2 \\
ECG storage capacity (min) & 60 & 66 & 59 \\
Diagnosed episodes & Asystole, Brady, Tachy, AF & Asystole, Brady, AF, HVR, SRD & Asystole, Brady, Tachy, AT, AF \\
Mean time of operation (years) & 2 & 4 & 3
\end{tabular}

AF - atrial fibrillation; AT - atrial tachycardia; HVR - high ventricular rate; SRD - sudden rate drop

b) Tasks of the cardiological centre

- Verification of indications for ICM implantation based on patient's medical records and discussing alternative methods of long-term ECG monitoring that are currently available

- ICM implantation

- ICM implantation in the CIED operating room; perioperative antibiotic prophylaxis in accordance with local standards is required; the procedure should be performed by a cardiologist experienced in CIED implantations

- ICM interrogation before discharge

- Schedule of follow-up visits

- Permanent remote monitoring in accordance with the standard of a given centre, but allowing everyday assessment of ICM report. If remote monitoring is not possible, additional visits should be scheduled to ensure that the device is interrogated every three months.

- Follow-up visit 3 months after implantation: assessment of the implantation site and ICM function

- Follow-up visits every 12 months

- Emergency visit in the case of recorded arrhythmia requiring a therapeutic decision

Observational studies show that the real-life diagnostic workup in Polish stroke units may not be sufficient to clearly determine if a given cryptogenic stroke may be classified as ESUS [6]. Therefore, if the patient has an opportunity of cost-free long-term ECG monitoring, it is especially important to start by making a diagnosis of ESUS as described in the previous section. It should also be considered to introduce an additional procedure of 48-72-hour ECG Holter monitoring or to equip stroke units with telemetric systems capable of automated detection of arrhythmias and adding it to the NHF list of procedures for stroke.

Follow-up visits should be performed by a cardiologist capable of addressing different cardiac conditions. It is important to provide the possibility of a telemetric rhythm control to ensure quick diagnosis of arrhythmias and immediate implementation of adequate treatment. ICM telemonitoring was an integral part of the diagnostic strategy used in clinical trials and therefore influenced the outcomes [28-30].

In our opinion, it is reasonable to maintain ICM and continue follow-up visits until battery depletion even in patients with AF detected by the device. This strategy is justified by the risk coincidence of clinically significant arrhythmias other than AF, and by the possibility of monitoring the AF treatment efficacy or diagnosing syncopes. The real costs of outpatient ICM visits may be considered similar to pacemaker visits, since they require a detailed overview and verification of all automatically detected and recorded episodes. The annual cost of telemedical follow-up is estimated to equal the cost of four pacemaker visits.

\section{Recommended conditions of arrhythmia monitoring with ICM following ESUS}

a) Neurological centres

- Stroke unit which admits at least 200 patients with ischaemic stroke a year

- Equipment and human resources allowing full standard diagnostic workup necessary to diagnose ESUS in each patient with cryptogenic ischaemic stroke

- Ability to provide follow-up at the hospital outpatient neurological clinic

b) Cardiological centres

- Ability to implant ICM within 4 weeks from the first information received from the cooperating stroke centre about a patient meeting neurological criteria of qualification

- Resources ensuring comprehensive care of AF patient, i.e. a team experienced in CIED procedures and electrophysiology (EP), CIED/EP operating theatre, on-site transthoracic and transoesophageal echocardiography, on-site access to prolonged ECG examination (7-day Holter, external arrhythmia recorders), CIED outpatient clinic, on-site general cardiological outpatient clinic

- Experience in remote monitoring of CIED

- Possibility of delivering a cardiological consultation on a 24 -hour basis 


\section{Health needs at the national level}

In order to estimate the health needs in the area of ICM implantation after ESUS, an additional analysis was conducted based on the material used in the study of Bembenek et al., making the following assumptions [6].

- The results presented in the publication of Bembenek et al. may be extrapolated to the general Polish population

- Inclusion criteria are the first-ever ischaemic stroke, age $\geq 65$ years, and left atrial enlargement

- Annual number of patients admitted to Polish stroke units for acute ischaemic stroke is 64,400

- At discharge from the stroke unit the patient is assessed as independent in daily activities ( $0-2$ points on the modified Rankin Scale)

In the basic variant (i.e. assuming that the proportion of ESUS patients will be equal to the proportion of patients with a certain ESUS diagnosis), the annual number of patients eligible for intense prolonged ECG monitoring may amount to 800 . In the maximum variant (i.e. including patients not classified as certain ESUS due to not having echocardiography), the annual number of cases may rise to 1,625.

Additionally, it must be emphasised that the data stored in the registry did not allow determination of the degree of left atrial enlargement. This means that the actual population size is probably less numerous. Moreover, not every patient will be willing to undergo a procedure that requires repeated visits to a specialised cardiological centre.

\section{Conclusion}

Adequate cardiological workup is one of the main factors determining the type of pharmacotherapy used in the secondary prevention of ischaemic stroke. In a large proportion of patients, standard diagnostics do not allow stroke aetiology to be determined. Therefore, there is a need for closer cooperation between stroke centres and cardiological centres and a need for systemic solutions that would facilitate such cooperation.

Research data indicates, that early initiation of prolonged ECG monitoring in a properly selected group of patients after embolic stroke of undetermined source could provide the chance of detecting atrial fibrillation, consequently reducing the risk of recurrence by timely introduction of oral anticoagulation.

Current technology allows long-term continuous ECG monitoring with the use of ICM. Due to high patient cost and the lack of dedicated financing from the NHF, this medical technology is not available to a Polish stroke patient. Nevertheless, considering the health benefits in the context of the whole population, efforts should be made to implement long term ECG monitoring after ESUS in reasonably selected patients.

Conflict of interest $M M F$ received consultancy, proctoring or lecturer fees from Medtronic, Abbott, Boston Scientific, Pfizer;
MK received lecturer fees from Boehringer Ingelheim, Ever Pharma, Bayer, travel grants for scientific conferences from Boehringer Ingelheim and Ever Pharma, consultancy fees from Medtronic; JK received lecturer fees from Medtronic, Biotronik, Abbott and proctoring fees from Medtronic; $P K$ received lecturer fees from Medtronic, Biotronik, Abbott; PM received honoraria from Medtronic, Biotronik, Abbott, Boston Scientific and investigator fees from Medtronic, Abbott; PP received lecturer fees from Medtronic, Abbott and grants from Medtronic; $D R$ received an honorarium for an advisory board meeting of Medtronic; IS received lecturer fees from Medtronic; AS received honoraria for advisory board meetings of Boehringer Ingelheim and advisory meetings of Bayer, Boehringer Ingelheim, Novartis, MERCK, lecturer fees from Bayer, Boehringer Ingelheim, Novartis, Polpharma, Bristol-Myers Squipp, Novartis, Biogen, Teva, Medtronic, MERCK; MS received consultancy and lecturer fees from Abbott, Adamed, Biotronik, Boehringer Ingelheim, Boston Scientific, Medtronic, Novartis, Pfizer and investigator fees from Biotronik, Medtronic, Zoll.

Ethics Ethical approval was not necessary for preparation of this article.

\section{References}

1. Kim AS, Cahill E, Cheng NT. Global Stroke Belt: Geographic Variation in Stroke Burden Worldwide. Stroke. 2015; 46(12): 3564-3570, doi: 10.1161/STROKEAHA.115.008226, indexed in Pubmed: 26486867.

2. Krishnamurthi RV, Feigin VL, Forouzanfar MH, et al. Global Burden of Diseases, Injuries, Risk Factors Study 2010 (GBD 2010), GBD Stroke Experts Group. Global and regional burden of first-ever ischaemic and haemorrhagic stroke during 1990-2010: findings from the Global Burden of Disease Study 2010. Lancet Glob Health. 2013; 1(5): e259-e281, doi: 10.1016/S2214-109X(13)70089-5, indexed in Pubmed: 25104492.

3. Mapa potrzeb zdrowotnych w zakresie lecznictwa szpitalnego dla Polski. Ministerstwo Zdrowia 2018. http://www.mpz.mz.gov. pl/mapy-szpitalne-ustawowe-2018/, dostęp 06. ; 12: 2018.

4. Adams HP, Bendixen BH, Kappelle LJ, et al. Classification of subtype of acute ischemic stroke. Definitions for use in a multicenter clinical trial. TOAST. Trial of Org 10172 in Acute Stroke Treatment. Stroke. 1993; 24(1): 35-41, indexed in Pubmed: 7678184.

5. Hart RG, Diener HC, Coutts SB, et al. Cryptogenic Stroke/ESUS International Working Group. Embolic strokes of undetermined source: the case for a new clinical construct. Lancet Neurol. 2014; 13(4): 429-438, doi: 10.1016/S1474-4422(13)70310-7, indexed in Pubmed: 24646875.

6. Bembenek JP, Karlinski MA, Kurkowska-Jastrzebska I, et al. Embolic strokes of undetermined source in a cohort of Polish stroke patients. Neurol Sci. 2018; 39(6): 1041-1047, doi: 10.1007/s10072-0183322-5, indexed in Pubmed: 29556872.

7. Li L, Yiin GS, Geraghty OC, et al. Oxford Vascular Study. Incidence, outcome, risk factors, and long-term prognosis of cryptogenic transient ischaemic attack and ischaemic stroke: a population-based study. Lancet Neurol. 2015; 14(9): 903-913, doi: 10.1016/S14744422(15)00132-5, indexed in Pubmed: 26227434.

8. Hart R, Pearce L. Antithrombotic Therapy To Prevent Stroke in Patients with Atrial Fibrillation. Annals of Internal Medicine. 2000; 132(10): 841, doi: 10.7326/0003-4819-132-10-200005160-00017. 
9. Ruff CT, Giugliano RP, Braunwald E, et al. Comparison of the efficacy and safety of new oral anticoagulants with warfarin in patients with atrial fibrillation: a meta-analysis of randomised trials. Lancet. 2014; 383(9921): 955-962, doi: 10.1016/S0140-6736(13)62343-0, indexed in Pubmed: 24315724.

10. López-López JA, Sterne JAC, Thom HHZ, et al. Oral anticoagulants for primary prevention, treatment and secondary prevention of venous thromboembolic disease, and for prevention of stroke in atrial fibrillation: systematic review, network meta-analysis and cost-effectiveness analysis. Health Technol Assess. 2017; 21(9): 1-386, doi: 10.3310/ hta21090, indexed in Pubmed: 28279251.

11. Hellwig S, Grittner U, Audebert H, et al. Non-vitamin K-dependent oral anticoagulants have a positive impact on ischaemic stroke severity in patients with atrial fibrillation. Europace. 2018; 20(4): 569-574, doi: 10.1093/europace/eux087, indexed in Pubmed: 28460024.

12. Boulanger JM, Lindsay MP, Gubitz G, et al. Canadian Stroke Best Practice Recommendations for Acute Stroke Management: Prehospital, Emergency Department, and Acute Inpatient Stroke Care, 6th Edition, Update 2018. Int J Stroke. 2018; 13(9): 949-984, doi: 10.1177/1747493018786616, indexed in Pubmed: 30021503.

13. Kirchhof $P$, Benussi $S$, Kotecha D, et al. ESC Scientific Document Group. 2016 ESC Guidelines for the management of atrial fibrillation developed in collaboration with EACTS. Eur Heart J. 2016; 37(38): 2893-2962, doi: 10.1093/eurheartj/ehw210, indexed in Pubmed: 27567408.

14. Hart RG, Catanese L, Perera KS, et al. Embolic Stroke of Undetermined Source: A Systematic Review and Clinical Update. Stroke. 2017; 48(4): 867-872, doi: 10.1161/STROKEAHA.116.016414, indexed in Pubmed: 28265016.

15. Diener HC, Easton JD, Granger CB, et al. RE-SPECT ESUS Investigators. Design of Randomized, double-blind, Evaluation in secondary Stroke Prevention comparing the EfficaCy and safety of the oral Thrombin inhibitor dabigatran etexilate vs. acetylsalicylic acid in patients with Embolic Stroke of Undetermined Source (RE-SPECT ESUS). Int J Stroke. 2015; 10(8): 1309-1312, doi: 10.1111/ijs.12630, indexed in Pubmed: 26420134.

16. Hart RG, Sharma M, Mundl H, et al. NAVIGATE ESUS Investigators. Rivaroxaban for Stroke Prevention after Embolic Stroke of Undetermined Source. N Engl J Med. 2018; 378(23): 2191-2201, doi: 10.1056/ NEJMoa1802686, indexed in Pubmed: 29766772.

17. Carmona-Puerta R, Castro-Torres Y. Atrial fibrillation and cryptogenic stroke. What is the current evidence? Role of electrocardiographic monitoring. J Arrhythm. 2018; 34(1): 1-3, doi: 10.1002/joa3.12016, indexed in Pubmed: 29721107.

18. Haeusler KG, Gröschel K, Köhrmann M, et al. Expert opinion paper on atrial fibrillation detection after ischemic stroke. Clin Res Cardiol. 2018; 107(10): 871-880, doi: 10.1007/s00392-018-1256-9, indexed in Pubmed: 29704214.

19. Haeusler KG, Tütüncü S, Schnabel RB. Detection of Atrial Fibrillation in Cryptogenic Stroke. Curr Neurol Neurosci Rep. 2018; 18(10): 66, doi: 10.1007/s11910-018-0871-1, indexed in Pubmed: 30090997.

20. Sanna T, Ziegler P, Crea F. Detection and management of atrial fibrillation after cryptogenic stroke or embolic stroke of undetermined source. Clinical Cardiology. 2018; 41(3): 426-432, doi: 10.1002/ clc.22876.

21. Widimsky P, Doehner W, Diener HC, et al. ESC Council on Stroke. The role of cardiologists in stroke prevention and treatment: position paper of the European Society of Cardiology Council on Stroke. Eur Heart J.
2018; 39(17): 1567-1573, doi: 10.1093/eurheartj/ehx478, indexed in Pubmed: 29020357.

22. Jonas D, Kahwati L, Yun J, et al. Screening for Atrial Fibrillation With Electrocardiography. JAMA. 2018; 320(5): 485, doi: 10.1001/ jama.2018.4190.

23. Chou PS, Ho BL, Chan YH, et al. Delayed diagnosis of atrial fibrillation after first-ever stroke increases recurrent stroke risk: a 5-year nationwide follow-up study. Intern Med J. 2018; 48(6): 661-667, doi: 10.1111/imj.13686, indexed in Pubmed: 29193638.

24. Powers WJ, Rabinstein AA, Ackerson T, et al. American Heart Association Stroke Council. 2018 Guidelines for the Early Management of Patients With Acute Ischemic Stroke: A Guideline for Healthcare Professionals From the American Heart Association/American Stroke Association. Stroke. 2018; 49(3): e46-e4e110, doi: 10.1161/ STR.0000000000000158, indexed in Pubmed: 29367334.

25. Grupa Ekspertów Sekcji Chorób naczyniowych Polskiego Towarzystwa Neurologicznego. Postępowanie w udarze mózgu. Neurol Neurochir Pol. 2012; 46: s1-116.

26. Steffel J, Verhamme P, Potpara TS, et al. ESC Scientific Document Group, ESC Scientific Document Group . The 2018 European Heart Rhythm Association Practical Guide on the use of non-vitamin $K$ antagonist oral anticoagulants in patients with atrial fibrillation. Eur Heart J. 2018; 39(16): 1330-1393, doi: 10.1093/eurheartj/ehy136, indexed in Pubmed: 29562325.

27. Mairesse GH, Moran P, Van Gelder IC, et al. ESC Scientific Document Group. Screening for atrial fibrillation: a European Heart Rhythm Association (EHRA) consensus document endorsed by the Heart Rhythm Society (HRS), Asia Pacific Heart Rhythm Society (APHRS), and Sociedad Latinoamericana de Estimulación Cardíaca y Electrofisiología (SOLAECE). Europace. 2017; 19(10): 1589-1623, doi: 10.1093/europace/eux177, indexed in Pubmed: 29048522.

28. Brachmann J, Morillo CA, Sanna T, et al. Uncovering Atrial Fibrillation Beyond Short-Term Monitoring in Cryptogenic Stroke Patients: Three-Year Results From the Cryptogenic Stroke and Underlying Atrial Fibrillation Trial. Circ Arrhythm Electrophysiol. 2016; 9(1): e003333, doi: 10.1161/CIRCEP.115.003333, indexed in Pubmed: 26763225.

29. Sanna T, Diener HC, Passman RS, et al. CRYSTAL AF Investigators. Cryptogenic stroke and underlying atrial fibrillation. N Engl J Med. 2014; 370(26): 2478-2486, doi: 10.1056/NEJMoa1313600, indexed in Pubmed: 24963567.

30. Ritter M, Kochhäuser S, Duning T, et al. Occult Atrial Fibrillation in Cryptogenic Stroke. Stroke. 2013; 44(5): 1449-1452, doi: 10.1161/ strokeaha.111.676189.

31. Muscari A, Bonfiglioli A, Faccioli L, et al. Usefulness of the MrWALLETS Scoring System to Predict First Diagnosed Atrial Fibrillation in Patients With Ischemic Stroke. Am J Cardiol. 2017; 119(7): 1023-1029, doi: 10.1016/j.amjcard.2016.12.009, indexed in Pubmed: 28153345.

32. Kwong C, Ling AY, Crawford MH, et al. A Clinical Score for Predicting Atrial Fibrillation in Patients with Cryptogenic Stroke or Transient Ischemic Attack. Cardiology. 2017; 138(3): 133-140, doi: 10.1159/000476030, indexed in Pubmed: 28654919.

33. Ricci B, Chang AD, Hemendinger M, et al. A Simple Score That Predicts Paroxysmal Atrial Fibrillation on Outpatient Cardiac Monitoring after Embolic Stroke of Unknown Source. J Stroke Cerebrovasc Dis. 2018; 27(6): 1692-1696, doi: 10.1016/j.jstrokecerebrovasdis.2018.01.028, indexed in Pubmed: 29501269.

34. Orlov MV, Ghali JK, Araghi-Niknam M, et al. Atrial High Rate Trial Investigators. Asymptomatic atrial fibrillation in pacemaker recipients: 
incidence, progression, and determinants based on the atrial high rate trial. Pacing Clin Electrophysiol. 2007; 30(3): 404-411, doi: 10.1111/j.1540-8159.2007.00682.x, indexed in Pubmed: 17367361.

35. Strickberger SA, Ip J, Saksena S, et al. Relationship between atrial tachyarrhythmias and symptoms. Heart Rhythm. 2005; 2(2): 125-131, doi: 10.1016/j.hrthm.2004.10.042, indexed in Pubmed: 15851283.

36. Choe WC, Passman RS, Brachmann J, et al. CRYSTAL AF Investigators. A Comparison of Atrial Fibrillation Monitoring Strategies After
Cryptogenic Stroke (from the Cryptogenic Stroke and Underlying AF Trial). Am J Cardiol. 2015; 116(6): 889-893, doi: 10.1016/j.amjcard.2015.06.012, indexed in Pubmed: 26183793.

37. Pürerfellner H, Sanders P, Sarkar S, et al. Adapting detection sensitivity based on evidence of irregular sinus arrhythmia to improve atrial fibrillation detection in insertable cardiac monitors. Europace. 2018; 20(FI_3): f321-f328, doi: 10.1093/europace/eux272, indexed in Pubmed: 29036652. 